\title{
Mechatronic Systems for Caregivers Support on the Context of Bedridden People Care
}

\author{
Solutions to better life
}

\author{
Karolina Bezerra ${ }^{1}$, José Machado ${ }^{1,2}$, Bruno Silva ${ }^{1}$, \\ Lídia Gonzalez ${ }^{1}$ \\ ${ }^{1}$ R\&D Metrics, School of Engineering \\ ${ }^{2}$ Dept. Mechanical Engineering, School of Engineering \\ University of Minho \\ Guimarães, Portugal \\ [karolceli@dem.uminho.pt,jmachado@dem.uminho.pt]
}

\author{
Vítor Carvalho ${ }^{3,5}$, Filomena Soares ${ }^{3,4}$, Demétrio \\ Matos ${ }^{5}$, Filipe Pereira ${ }^{1}$ \\ ${ }^{3}$ R\&D Algoritmi, School of Engineering \\ ${ }^{4}$ Dept. Industrial Electronics, School of Engineering \\ University of Minho \\ Guimarães, Portugal \\ ${ }^{5}$ Polytechnic Institute of Cávado and Ave \\ Barcelos, Braga \\ [vcarvalho@ipca.pt, fsoares@dei.uminho.pt, \\ dmatos@ipca.pt]
}

\begin{abstract}
With the increasing number of aged people, especially in developed countries, Wellbeing solutions have became an important subject to be explored and developed. Currently, as specialized Institutions in geriatric care cannot cope with the increasing requests for support of quality of life, patients have to remain at their homes having as caregiver the other member of the couple or a member of close family. A solution for supporting the caregiver, during assisting the bedridden person with some basic tasks as eating, taking a bath and/or hygiene care is of utmost importance. This paper presents an overview for supporting the caregiver on providing the basic needs for bedridden persons. From safety needs to repositioning and hygiene care of bedridden persons are taken into account, by developing specific mechatronic devices for supporting and helping caregivers on those tasks. The proposed mechatronic systems must, ideally, reduce the number of caregivers and the amount of spent and needed effort.
\end{abstract}

Keywords-Health care for older people, Assisted Technology, Health care at home, Mechanical design and Mechatronics systems.

\section{INTRODUCTION}

For many families the easiest way to give their bedridden some quality of life is institutionalizing them in nursing homes, hospitals, adult daycare centers or hospices. Although in most of times is the best solution, it has the disadvantages of being costly and overflowing with admission requests. Furthermore, there are not enough trained professionals to take proper care of the elder. Because of this many elders have to stay with their families and be taken care by them. In some cases, the one who takes care is the life partner who is about the same age as the one who needs special attention. As we can infer these individuals don't have much strength to deal with the bedridden and as consequence is possibly to observe a decay of physical and psychological health of both of them $[1,2]$.

The available solutions in the marketfor better movement and repositioning of the elderly are deficient and lacking. The current domestic solutions are less costly and less sturdy versions of the ones we can find in the hospital. These devices only meet one need and in order to do other tasks it is necessary to combine features of different devices and physical aid from a caregiver. Although it is a problem that many public and private organizations are aware, there is no development of better applications. The main motivation behind this work is to develop a mechanism that contemplates three of five domains specified for developing assisting devices:

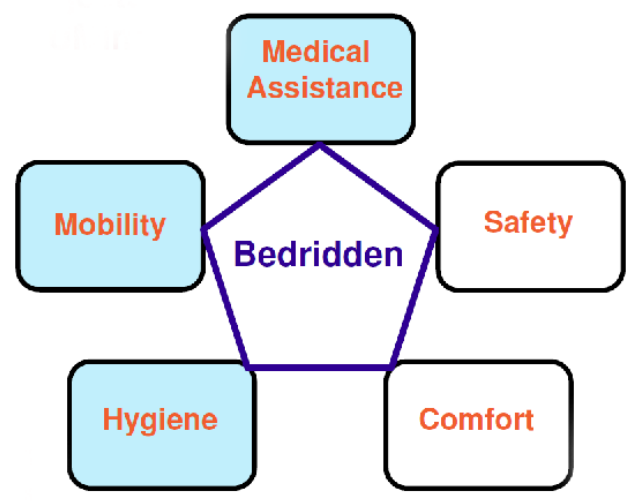

Fig. 1 Scheme of domains for assistive devices 


\section{DOMAINS PROJECTS}

\section{A. Medical Assitance}

\section{1) Medical Care Terminal}

To allow the better of taking care of bedridden people was developed a proposed system is based on a medical care terminal (MCT) and a portable mechatronic system. This integrate system pretends supply to collect physiological data of the patient and allows a remote medical interface, creating a new perspective for doctors, withmedical instructions to prescribe to the patient. The environment variables are included to be monitored by the system, the process of this system is shows in Figure 2[3].

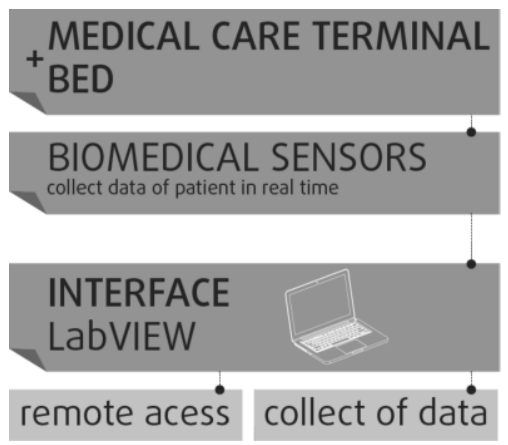

Fig. 2 Schematic MCT process of the system [3]

The medical care terminal (MCT) system part pretended to have high quality biomedical sensors that perform the analyses in real time through an embedded system that enables the operation of biomedical sensors.

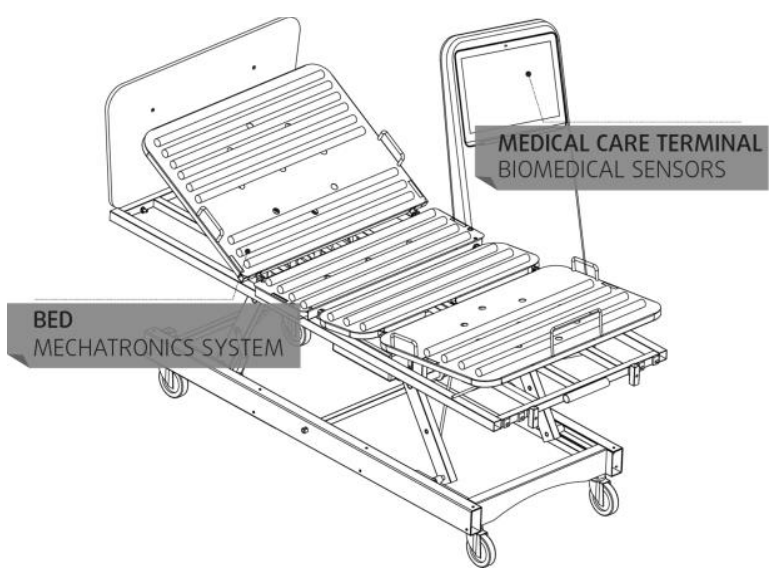

Fig. 3 Example of the integrated system (Bed with MCT) [3]

This system becomes versatile because it does not depend on the application in which it will be used. In this case, a biomedical indication can be used by six different connectivity options: Wi-Fi, 3G, General Packet Radio Service (GPRS) Bluetooth, 802.15.4 and ZigBee. The Medical Care Terminal (MCT) is divided in three parts, namely: Acquisition Software, System Hardware and User Interface [3]. a) Biomedical Sensors

In Figure 4, the biomedical sensor, responsible for measuring respiration in the integrated MCT system, can be identified. The purpose of this method is to measure values and collect information about the values obtained in the patient's mobilization, with the main objective of providing the preliminary and diagnostic data to the respective responsible.

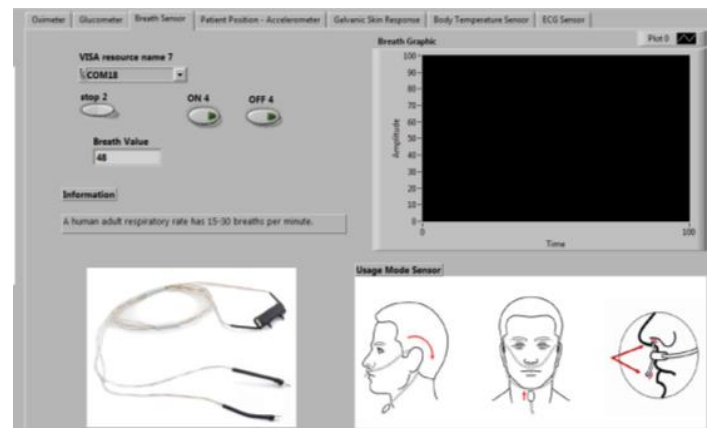

Fig. 4 LabVIEW Interface [3]

\section{b) Environmental Sensor}

The interface developed in the Labview has an important part, shown in Figure 5, which shows the operation of the environmental sensor, specification is used a light sensor that is able to include in the interface a led light that aims to alert when there is a lack of Light in the care environment[3].

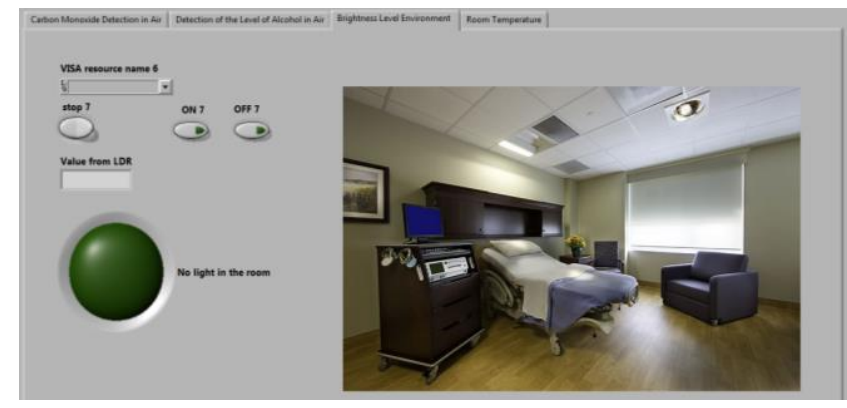

Fig. 5 LabVIEW Interface for environmental Sensors [3]

c) Remote Communication 
Through a webcam that is part of the integrated MCT system (Figure 6), it is able to provide remote patient communication with a physician or specialist; The developed application allows the patient to obtain basic care information in emergencies.

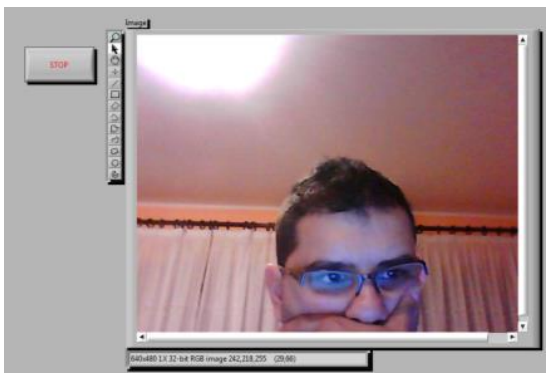

Fig. 6 Remote Communication [3]

This system integrated in the MCT has as main objective the development of a mechatronical system capable of giving a higher quality of life to people in bed. Provides greater freedom and speed by providing real-time monitoring of physiological data and care environment conditions, it may occur through remote monitoring and storage of in-database information[3].

\section{B. Mobility}

\section{1) Handling}

To help a daily hygiene routinebedridden elderly people (BEP) was developed a mechanical system is to facilitate the movement and repositioning of the bedridden individual. It is intended to be used with the individual is lying down, the system must be able to move him/her around providing comfort and reducing the caregiver's effort when handling.

A solution has been designed for easy handling, therefore, as can be seen in figure 7 , the apparatus is a low profile conveyor modulus that is inserted underneath the bed. It has an ability to extend on both sides of the base.

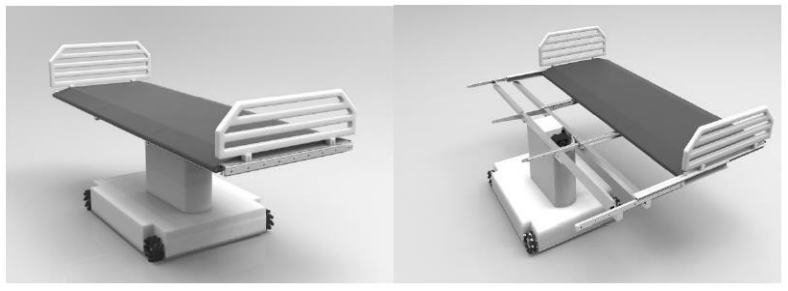

Fig. 7 3D CAD Rendering of solution [4]

The possibility of placing the drive mechanism of the conveyor is of extreme importance so that the device is capable of being used on the user.

It was defined that no space isavailable for the placement of such components on the sides of conveyer module where the noseroller or knife-edge will be place since these are of low height. One should pay attention, in the place where patients are positioned, should not be placed above any part of the plan. In case any component underneath the transported module compromises the ability of the module to function in the activities of advancing at the same time as the low profile.

In the specifications, the profile of the conveyor structure has $47 \mathrm{~mm}$,it can be considered a low profile solution. In Figure 8, noneparts areencumbering the space over and under the conveyor modules [4].



Fig. 8 Example of Transmission [4]

Some gaps were solved through the implementation of conveyor belts that served to drive less slack.In figure 8 , we can identify the rotary pulleys indicated by the red arrows that run the length of the conveyor module and are fixed to a shaft.

In Figure 8 characterized "Attachment opening", the path of these belts describe a smaller profile then the centered conveyor belt in order to create the opening. Because then getup-and-go two smaller tensioned belts on each side.

The main of this opening is to provide an attachment location to the telescopic rail and thus creating a seamless joint between modules such that the break in beltmaterial is negligible[4].

The final aspect can be seen in Figure 9. Is important mentioned the construction and components, they are very primary available in any local hardware store. The trolley discussed in the previous chapter is in fact the conveyer module and is represented here by the grey model of the conveyer structure [4]. 


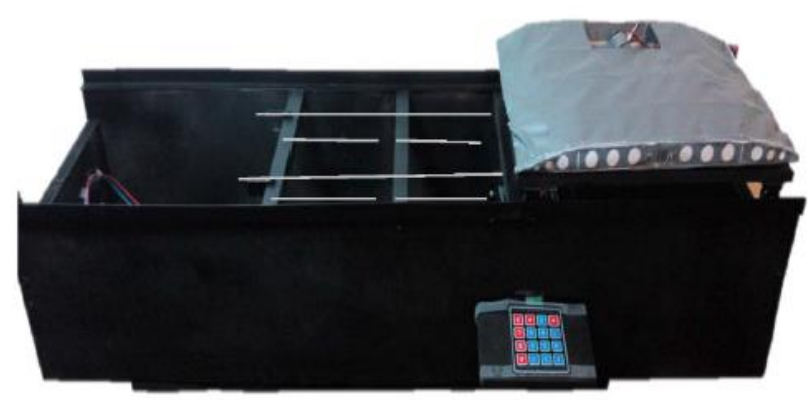

Fig. 9 Developed prototype

\section{Hygiene}

1) Bath

To continue with solutions to help beddriden people was developed this part presents the conceptual design of a mechatronic system especially devoted to the assistance during the bath of BEP. In this project are the new parameters for the development of bath equipment, such as the possibility of reducing the number of caregivers to only one auxiliary, and another parameter is to reduce a system manipulation complexity - considered to be used by a person Elderly caregiver. In the development stage of this project, it was crucial to have meetings with caregivers and visits to rehabilitation centers and hospitals to obtain information about the work they perform in daily care, in order to know the levels of difficulties and their real needs for development of the mechatronic system for the bath.

\section{a) Proposed concept}

This proposal for a new system has been developed in the ALL context, specifically in the area of daily care of people in bed. The mechatronic system promises to facilitate the task of caregivers and better the problems related to the activity of the Bath. The system competes not only with the task of cleaning,but comes to become a portable project to be used in homes, whose technology allows the monitoring of the operation avoiding cases of accidents (SCB)[5].

Environment variables can also be monitored by the system. The schematic is presented in Figure 10.

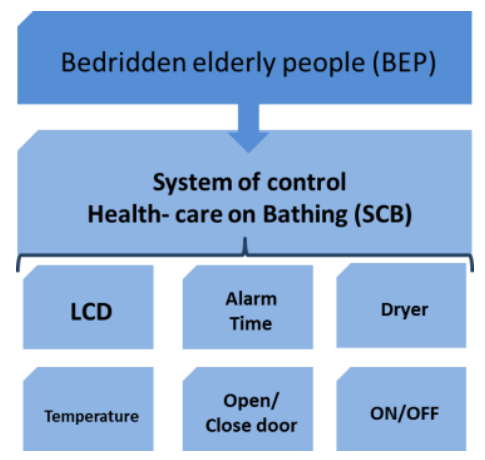

Fig. 10 Schematic of the proposed system [5]
The attention taken related to the health conditions of the elderly, the design of the system aims to solve problems or some, in the bathing activity. Activities that involve the transportation, cleaning and drying of the user. The BED comes to be an innovative system that integrates technologies creating a bath variable control system (SCB).

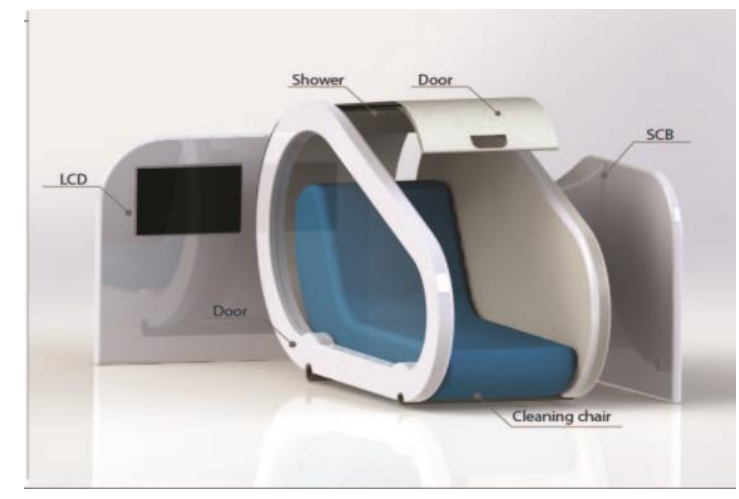

Fig. 11Final concept proposed [5]

In a perspective of promoting the best quality of life of bedridden, the integrated system has the ability to monitor bathing activities, such as water temperature.

To constitute a structure of equipment for the bath of PVC (Polyvinyl chloride). As your specifications show the result of a superficial lodging and non-irritable integration with skin contact.

\section{b) Funcionalities}

The mechatronic system has functional parts, being: a central door, a lateral (transparent) and wheels. The integral parts become the independent system, therefore they are modular with connection to one which facilitates the use and transfer of the camping into the system, safely and comfortably.

The SCB system is part of a safety system that seeks to control the movements through a starter motor and quick closing mechanisms, allowing to interrupt any operation. This is possible by the fact that there are position sensors implemented that ensure correct fit in the elderly within the system. In addition, the system is controlled and monitored through the external monitor.

In figure 12 can be identify the bed interface designed to be connected to the bath system, it is a transport system to transfer the patient safely and comfortably to into the bath system. This part has a mechanical scissor system that provides bed elevation through uses a motor and a linear actuator[5]. 


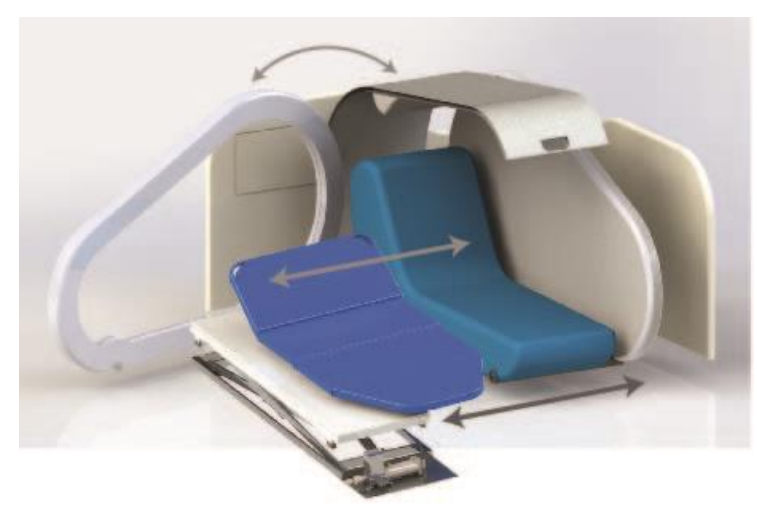

Fig. 12 Demonstration of use [5]

The system has a functional parts: two types of doors (Figure 12). The first door is used to close of the ambience when the patient is inside,. The second door is lateral and is used to facilitate people transfer; it is made in transparent material to allow the interaction between caregivers and elders during bath. From the touch control the doors open, this control functions of the SCB are inserted into the panel that connects directly with the BED.

\section{c) Shower}

The shower system is a adapted to a specific part to clean the users inside of system. It's important part of mechatronic system was design specifically to this project and is controlled over a Human Machine Interface (HMI) from the LCD monitor.

The control of water is done considering the measurement of a certain level; when full, it is enough for 2 showers a day. On the bottom of the SCB, it's located the water supply from internal storage of the bath system[5].

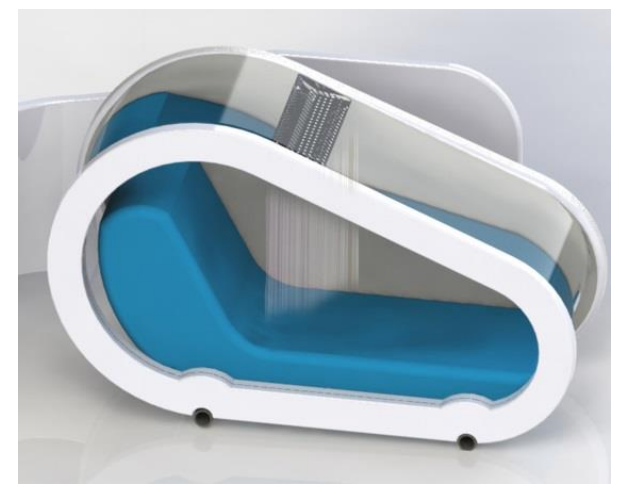

Fig. 12 Shower Demonstration [5]

\section{CONCLUSIONS}

This article presents the work done by an interdisciplinary research group. The aim of this ongoing research is to develop various solutions to support the continued care of finished patients. Developments are fostered at the present moment in the area of medical assistance, mobility and hygiene for bedridden patients (BEP).

The first concept aims at supporting the caregiver to monitor in real time the health state of a patient by collecting biomedical data in order to provide remote medical help. The data collection system is integrated in the biomedical module of a mechatronic system. It presents various advantages, such as:

\section{- User-friendly interface}

- Communication with central medical unit is facilitated for a better control of the patient

- Close hardware/ software integration for fast response

- Multiple sensors are integrated in one application

- Biomedical data of patient and environmental data are collected to monitor the patient's comfort.

Research on the second concept, called the "transforming bed", showed its viability but further studies must be performed. The implementation of previously described system constraints has to be validated. Kinematic concepts were already developed. It was aimed to design a mechanical system that facilitates the movement and repositioning of the BEP.

The third concept is a mechatronic system that allows accessibility, privacy and safety for the bathing area. Moreover, the monitoring system can be equipped with further functions adjustable to specific patient's needs in order to control tasks that the caregiver may perform. The mechanical unit has to be further developed to enable manual usage in case failure occurs in mechatronic components.

\section{Acknowledgment}

This work has been supported by COMPETE: POCI-01-0145FEDER-007043 and FCT - "Fundação para a Ciência e Tecnologia" within the Project Scope: UID/CEC/00319/2013.

\section{References}

[1] H. Henna, A, Judith, Care recipients' and family members' perceptions of quality of older people care: a comparison of home-based care and nursing homes, Journal of Clinical Nursing, 20(9-10), 2011, 1423-35.

[2] L. Tove, I. Bolmsjö, I. R. Hallberg. Standing Guard-being a Relative to a Hospitalised, Elderly Person. Journal of Aging Studies, 20(2), 2006, 133-4.

[3] P. Filipe, C. Victor, S. Filomena, M. José; B. Karolina, S. Rui; M. Demétrio. Development of a Mechatronic System for Bedridden People Support. Proceedings of MECAHITECH'13 - 5th International Conference on Innovations, Recent Trends and Challenges in Mechatronics, Mechanical Engineering and New High-Tech Products Development, Bucharest, 2013.

[4] B. Silva,M. José, S. Filomena, C. Vítor, M. Demétrio, B. Karolina.The Conceptual Design of a Mechatronic System to Handle Bedridden Elderly Individuals. Sensors, 16, 2016, 5: 725 - 725.

[5] B. Karolina, M. José, C. Vítor, S. Filomena, S. Bruno, M. Demétrio, L. Celina. System for Assistance on Bath of Bedridden Elderly People. Proceedings of ASME 2014 International Mechanical Engineering 
Recent Innovations in Mechatronics (RIiM) Vol. 4. (2017). No. 1.

DOI: 10.17667/riim.2017.1/6.

Congress and Exposition (IMECE204), Montreal, Quebec, Canada, 5, 2014, 14-20. 